\title{
SPECTRAL RECONSTRUCTION OF PIECEWISE SMOOTH FUNCTIONS FROM THEIR DISCRETE DATA
}

\author{
AnNe GelB ${ }^{1}$ AND EITAN TADMOR ${ }^{2}$
}

\begin{abstract}
This paper addresses the recovery of piecewise smooth functions from their discrete data. Reconstruction methods using both pseudo-spectral coefficients and physical space interpolants have been discussed extensively in the literature, and it is clear that an a priori knowledge of the jump discontinuity location is essential for any reconstruction technique to yield spectrally accurate results with high resolution near the discontinuities. Hence detection of the jump discontinuities is critical for all methods. Here we formulate a new localized reconstruction method adapted from the method developed in Gottlieb and Tadmor (1985) and recently revisited in Tadmor and Tanner (in press). Our procedure incorporates the detection of edges into the reconstruction technique. The method is robust and highly accurate, yielding spectral accuracy up to a small neighborhood of the jump discontinuities. Results are shown in one and two dimensions.
\end{abstract}

Mathematics Subject Classification. 42A10, 42A50, 65T40.

Received: July 19, 2001.

\section{BRIEF INTRODUCTION}

Let $f(x)$ be a $2 \pi$-periodic piecewise smooth function. Suppose we are given the values $f\left(x_{j}\right)$ at the grid points $x_{j}=-\pi+j \Delta x, j=0, \cdots, 2 N$, and $\Delta x:=\frac{2 \pi}{2 N+1}$. We wish to recover $f(x)$ at some intermediate grid point values, say, $x=x_{j+\frac{1}{2}}$. There is extensive literature on recovering piecewise smooth functions both from pseudo-spectral coefficients (e.g. [9]) as well as by physical space interpolation $[10,12]$. Essential to any reconstruction approach is the ability to locate the jump discontinuities of $f(x)$ from its discrete data points. While many edge detection techniques have been extensively studied, including those using the locally based wavelets and finite difference methods, our goal here is to retrieve exponential accuracy for the recovery of piecewise smooth functions. Exponential accuracy is a main feature of spectral representations of smooth data, and appropriate reconstruction procedures are required for exponential recovery of piecewise smooth data. Since spectral methods are global, these recovery procedures differ from those of the polynomially accurate, local finite difference and wavelets based methods. Our recovery procedure consists of detection of edges in spectral data followed by exponentially accurate reconstruction procedure to recover the smooth parts in between those edges.

Keywords and phrases. Edge detection, nonlinear enhancement, concentration method, piecewise smoothness, localized reconstruction.

1 Department of Mathematics, P.O. Box 871804, Arizona State University, Tempe, AZ 85287-1804, USA.

e-mail: ag@math.la.asu.edu

2 Department of Mathematics, UCLA, Los Angeles, CA 90095-1555, USA. e-mail: tadmor@math.ucla.edu 
Here we construct a new localized reconstruction method, adapted from the method developed in [10] and recently revisited in [12], where a physical space interpolation is used to approximate $f(x)$. The original localized reconstruction method is reviewed in Section 2. The new method consists of first detecting the neighborhoods of the jump discontinuities of $f(x)$, considered in Section 3, and then pinpointing the exact jumps by nonlinear enhancement, discussed in Section 4. A highly accurate and robust scheme is devised, yielding spectral accuracy up to a small neighborhood of the jump discontinuities. Numerical results in both one and two dimensions are produced.

\section{RECOVERING INTERMEDIATE POINT VALUES FROM DISCRETE DATA}

\subsection{Local reconstruction for piecewise smooth functions in one dimension}

Let $f(x)$ be a $2 \pi$-periodic piecewise smooth function with a single jump discontinuity at $x=\xi$. Given its discrete grid values $f\left(x_{j}\right)$ at the $2 N+1$ equidistant points, $x_{j}:=-\pi+j \Delta x$, with $\Delta x:=\frac{2 \pi}{2 N+1}$, we wish to recover $f(x)$ at the intermediate grid points, $w=x_{j+\frac{1}{2}}$. Recall the standard pseudo-spectral approximation

$$
I_{N}[f](w)=\sum_{k=-N}^{N} \tilde{f}_{k} \mathrm{e}^{i k w},
$$

where the pseudo-spectral coefficients are defined as

$$
\tilde{f}_{k}=\frac{1}{2 N+1} \sum_{j=0}^{2 N} f\left(x_{j}\right) \mathrm{e}^{-i k x_{j}} .
$$

Since the accuracy of (2.1) depends on the global smoothness of $f$, the pointwise errors $\left|I_{N}[f](w)-f(w)\right|$ are large even if $f(\cdot)$ is smooth in the local neighborhoods of $w$ 's away from the discontinuity at $x=\xi$.

In [10], the authors developed a filtering method which recovers the pointwise values of a piecewise smooth function from the information contained in its (pseudo-) spectral approximation. By employing a localized regularization kernel, one recovers spectral accuracy at intermediate grid point values away from the discontinuities. Moreover, an adaptive version of this filtering kernel introduced in [12], yields an increasingly higher order throughout the smooth regions, with exponential accuracy at their interiors. The reconstruction depends solely on the local smoothness of $f$. We assume that $f$ is piecewise smooth in the sense of being $C^{2}$ except for finitely many jump discontinuities. Furthermore, the control of the localization by an appropriate cut-off function is carried out explicitly in the physical space, and hence the pseudo-spectral coefficients need not be computed. The basic ingredients, taken from [10,12], are summarized below.

Let $\rho(\zeta)$ be a $C^{\infty}$ function vanishing outside the interval $(-1,1)$ and normalized so that $\rho(0)=1$. As an example of such a $C^{\infty}$ cut-off function, one may choose

$$
\rho(\zeta) \equiv \rho_{\alpha}(\zeta)= \begin{cases}\mathrm{e}^{\frac{\alpha \zeta^{2}}{\zeta^{2}-1}}, & \text { if }|\zeta|<1 \\ 0 & \text { otherwise }\end{cases}
$$

depending on an arbitrary free $\alpha>0$ (the computations reported in Sect. 5 below are carried out with $\alpha=10$ ). Following [10], we introduce the regularization kernel $\Psi^{\theta, p}(w)$ defined as

$$
\Psi^{\theta, p}(w):=\frac{1}{\theta} \rho_{\alpha}\left(\frac{w}{\theta}\right) D_{p}\left(\frac{w}{\theta}\right)
$$


where $D_{p}(w)$ is the usual Dirichlet kernel

$$
D_{p}(w):=\frac{1}{2 \pi} \sum_{k=-p}^{p} \mathrm{e}^{i k w} \equiv \frac{1}{2 \pi} \frac{\sin \left(p+\frac{1}{2}\right) w}{\sin \frac{w}{2}} .
$$

Here $p=N^{\beta}$, with $\theta, \beta \in[0,1]$ are free parameters at our disposal. An optimal choice corresponding to $\beta=1$ was recently worked out in [12, Th. 3.2], yielding $p \sim \theta N$. Thus, we focus attention on the choice of $\theta$ which is outlined below.

Suppose for now that the spectral approximation of $f(x)$ is given by the continuous Fourier coefficients,

$$
S_{N}[f](w)=\sum_{k=-N}^{N} \hat{f}_{k} \mathrm{e}^{i k w}, \quad \hat{f}_{k}=\frac{1}{2 \pi} \int_{-\pi}^{\pi} f(x) \mathrm{e}^{-i k x} \mathrm{~d} x
$$

The spectral approximation can be smoothed via convolution with the regularization kernel forming

$$
S_{N}^{\Psi}[f](w)=S_{N} * \Psi^{\theta, p}(w)=\int_{-\pi}^{\pi} S_{N}(x) \Psi^{\theta, p}(w-x) \mathrm{d} x
$$

Since $\Psi^{\theta, p}(w)$ is supported in the $\theta$ neighborhood of the origin, the convolution acts as a window allowing only the smooth neighborhoods of $f$ to be considered in the approximation of $S_{N}^{\Psi}[f](w)$.

The discrete analog for (2.7) recovered from the partial sum (2.1) can be directly computed in physical space as

$$
I_{N}^{\Psi}[f](w)=I_{N} * \Psi^{\theta, p}(w)=\Delta x \sum_{j=0}^{2 N} f\left(x_{j}\right) \Psi^{\theta, p}\left(w-x_{j}\right), \quad \Delta x=\frac{2 \pi}{2 N+1}
$$

Due to the localizing effect of $\Psi^{\theta, p}(w)$, only the neighboring grid values $\left|x_{j}-w\right| \leq \theta$ are utilized. If $f$ is smooth near $w$, say in the neighborhood $[w-\theta, w+\theta]$, then $(2.8)$ becomes

$$
I_{N}^{\Psi}[f](w)=\frac{\Delta x}{2 \pi \theta} \sum_{\left|x_{j}-w\right| \leq \theta} f\left(x_{j}\right) \rho\left(\frac{w-x_{j}}{\theta}\right) \frac{\sin \left(p+\frac{1}{2}\right) \frac{w-x_{j}}{\theta}}{\sin \frac{1}{2} \frac{w-x_{j}}{\theta}} .
$$

It was shown in [10] that (2.7)-(2.9) recovers the point values of $f(x)$ with spectral accuracy, i.e., an arbitrarily fast convergence rate is achieved as long as $f$ remains smooth in the $\theta$-neighborhood of $w$. Moreover, the convergence is exponential provided $\theta$ is chosen so that $f \in C^{\infty}[w-\theta, w+\theta]$, [12]. Furthermore (2.9) is very cost effective since the pseudo-spectral coefficients (2.2) need not be computed.

\subsection{Local reconstruction for functions with several jump discontinuities in one dimension}

In order to recover a general piecewise smooth function $f(x)$ on $[-\pi, \pi]$ admitting several jump discontinuities, $\left\{\xi_{k}\right\}_{k=1}^{M}$, we choose $\theta=\theta(w)=\min \left\{\theta_{\text {left }}, \theta_{\text {right }}\right\}$, measuring the distance of $w$ to the nearest discontinuity, $\theta_{\text {left }}=w-\xi_{k}$ and $\theta_{\text {right }}=\xi_{k+1}-w$. Then (2.9) is performed in symmetric intervals only in the smooth regions $\xi_{k}<w<\xi_{k+1}, k=1, \cdots, M-1$, as

$$
I_{N}^{\Psi}[f](w)=\frac{\Delta x}{2 \pi \theta} \sum_{\left|x_{j}-w\right|<\theta} f\left(x_{j}\right) \rho\left(\frac{w-x_{j}}{\theta}\right) \frac{\sin \left(p+\frac{1}{2}\right) \frac{w-x_{j}}{\theta}}{\sin \frac{1}{2} \frac{w-x_{j}}{\theta}} .
$$

Restricting each $\theta(w)$ in this way ensures not only that the jump discontinuities $\left\{\xi_{k}\right\}_{k=1}^{M}$ are not crossed, but also that the largest (symmetric) region of smoothness is used in the approximation. However, as we 
approach the discontinuities there are apparently not enough points in the symmetric interval to perform the approximation (2.10). Therefore, when $w$ is within a small neighborhood of $\xi_{k}$ we adjust the parameter $\theta$ so that (2.10) acts simply to locally smooth the function at the discontinuities. More specifically, we define $\theta=\theta(w)=\max \left\{\min \left(\theta_{\text {left }}, \theta_{\text {right }}\right), \epsilon\right\}$, where $\epsilon$ is a small fixed parameter defining the neighborhood of each $\xi_{k}$. In this way the Gibbs phenomenon can be completely avoided. As mentioned above, (2.10) is very cost effective, since the pseudo-spectral approximation (2.1) need not be computed. High accuracy is obtained everywhere outside a small neighborhood $\epsilon$ of the jump discontinuities.

Critical to the reconstruction procedure $(2.10)$ is the knowledge of the jump discontinuities $\left\{\xi_{k}\right\}_{k=1}^{M}$. The procedure used to determine these discontinuities will be discussed in Section 3.

\subsection{Local reconstruction of piecewise smooth functions in two dimensions}

Suppose we are given the discrete grid values $f\left(x_{i}, y_{j}\right)$, defined at the equidistant points, $x_{i}:=-\pi+i \Delta x$, $y_{j}=-\pi+j \Delta y$, for a $2 \pi$-periodic piecewise smooth function $f(x, y)$, i.e., both $f(x, \cdot)$ and $f(\cdot, y)$ are $C^{2}$ except for finitely many jump discontinuities. For simplicity assume the same number of points are used in each direction so that $\Delta x=\Delta y:=\frac{2 \pi}{2 N+1}$. We wish to recover $f(x, y)$ at some intermediate grid point values, say, $\bar{x}=x_{i+\frac{1}{2}}$ and $\bar{y}=y_{j+\frac{1}{2}}$. A jump discontinuity is identified by its enclosed grid cell, $x_{i} \leq \xi \leq x_{i+1}, y_{j} \leq \eta \leq y_{j+1}$, and is characterized by the asymptotic statements

$$
\begin{aligned}
& f\left(x_{i+1}, y\right)-f\left(x_{i}, y\right)= \begin{cases}{[f](\xi, y)+\mathcal{O}(\Delta x)} & \text { for } i=i_{\xi}: \xi \in\left[x_{i}, x_{i+1}\right], \\
\mathcal{O}(\Delta x) & \text { for other } i^{\prime} s \neq i_{\xi} .\end{cases} \\
& f\left(x, y_{j+1}\right)-f\left(x, y_{j}\right)= \begin{cases}{[f](x, \eta)+\mathcal{O}(\Delta y)} & \text { for } j=j_{\eta}: \eta \in\left[y_{j}, y_{j+1}\right], \\
\mathcal{O}(\Delta y) & \text { for other } j^{\prime} s \neq j_{\eta} .\end{cases}
\end{aligned}
$$

Let us assume that the jump discontinuities

$$
\xi_{i}(\bar{y}), i=1, \cdots M_{x}(\bar{y}), \quad \eta_{j}(\bar{x}), j=1, \cdots M_{y}(\bar{x}),
$$

are known at the fixed intermediate grid point values, $\bar{x}$ and $\bar{y}$, which represent the discrete data points for the continuous function $f(x, y)$. We define the smooth neighborhoods for the recovery points, $\bar{x}(\bar{y})$ and $\bar{y}(\bar{x})$, as

$$
\begin{aligned}
\xi_{\text {left }}(\bar{y}) & =\bar{x}(\bar{y})-\xi_{i}(\bar{y}), \\
\xi_{\text {right }}(\bar{y}) & =\xi_{i+1}(\bar{y})-\bar{x}(\bar{y}), \\
\eta_{\text {left }}(\bar{x}) & =\bar{y}(\bar{x})-\eta_{j}(\bar{x}), \\
\eta_{\text {right }}(\bar{x}) & =\eta_{j+1}(\bar{x})-\bar{y}(\bar{x}),
\end{aligned}
$$

where $i=1, \cdots, M_{x}(\bar{y})-1$ and $j=1, \cdots, M_{y}(\bar{x})-1$. Applying the localized reconstruction method (2.10) in two dimensions, we have for each smooth region $\bar{x} \in\left[\xi_{i}(\bar{y}), \xi_{i+1}(\bar{y})\right]$ and $\bar{y} \in\left[\eta_{j}(\bar{x}), \eta_{j+1}(\bar{x})\right]$

$$
\begin{array}{r}
I_{N}^{\Psi}[f](\bar{x}, \bar{y})=\frac{\Delta x \Delta y}{2 \pi \xi_{x}(\bar{y}) \cdot 2 \pi \eta_{y}(\bar{x})} \sum_{\left|x_{i}-\bar{x}\right|<\xi(\bar{y})} \sum_{\left|y_{j}-\bar{y}\right|<\eta(\bar{x})} f\left(x_{i}, y_{j}\right) \rho\left(\frac{\bar{x}-x_{i}}{\xi_{x}(\bar{y})}\right) \rho\left(\frac{\bar{y}-y_{j}}{\eta_{y}(\bar{x})}\right) \\
\times \frac{\sin \left(\left(p+\frac{1}{2}\right) \frac{\bar{x}-x_{i}}{\xi_{x}(\bar{y})}\right)}{\sin \frac{1}{2}\left(\frac{\bar{x}-x_{i}}{\xi_{x}(\bar{y})}\right)} \cdot \frac{\sin \left(\left(p+\frac{1}{2}\right) \frac{\bar{y}-y_{i}}{\eta_{y}(\bar{x})}\right)}{\sin \frac{1}{2}\left(\frac{\bar{y}-y_{i}}{\eta_{y}(\bar{x})}\right)}
\end{array}
$$

where $\xi_{x}(\bar{y})=\max \left\{\min \left(\xi_{\text {left }}(\bar{y}), \xi_{\text {right }}(\bar{y})\right), \epsilon(\bar{y})\right\}$ and $\eta_{y}(\bar{x})=\max \left\{\min \left(\eta_{\text {left }}(\bar{x}), \eta_{\text {right }}(\bar{x})\right), \delta(\bar{x})\right\}$. As in the one-dimensional case from Section 2.1, $\epsilon(\bar{y})$ and $\delta(\bar{x})$ define the small local neighborhoods of $\xi_{i}(\bar{y})$ and $\eta_{j}(\bar{x})$ for 
which the approximation (2.12) is locally smoothed. Hence $f(\bar{x}, \bar{y})$ is approximated by employing the grid values in the largest symmetric interval within a smooth region up to a small neighborhood of the jump discontinuities. High accuracy is ensured away from the jump discontinuities while the Gibbs phenomenon is completely avoided. Furthermore, the localized reconstruction method (2.12) is done completely in physical space, making it very cost effective. The error analysis is conducted in the same way as the one-dimensional case [10], and is not repeated here.

Once again we see that the high accuracy of (2.12) is critically dependent upon the correct identification of the jump discontinuities, $\left\{\xi_{i}(\bar{y})\right\}_{i=1}^{M_{x}(\bar{y})}$ and $\left\{\eta_{j}(\bar{x})\right\}_{j=1}^{M_{y}(\bar{x})}$. We turn now to discuss the procedure which detects the locations of these discontinuities.

\section{Edge DeteCtion From DiscRETE DATA}

\subsection{Detection based on concentration factors}

Let $f(x)$ be a $2 \pi$-periodic piecewise smooth function with a single jump discontinuity at $x=\xi$, whose value is defined as $[f](\xi)=f\left(\xi^{+}\right)-f\left(\xi^{-}\right)$. Suppose for now that we are given the continuous Fourier coefficients

$$
\hat{f}_{k}=\frac{1}{2 \pi} \int_{-\pi}^{\pi} f(x) \mathrm{e}^{-i k x} \mathrm{~d} x
$$

We wish to detect the jump discontinuity $x=\xi$. To this end, let us recall the general concentration sum for detecting the edges proposed in $[6]$ and $[7]$ :

$$
\tilde{S}_{N}^{\sigma}[f](x):=\pi i \sum_{k=-N}^{N} \operatorname{sgn}(k) \sigma\left(\frac{|k|}{N}\right) \hat{f}_{k} \mathrm{e}^{i k x} .
$$

Integration by parts tells us that $\hat{f}_{k} \sim[f](\xi) \frac{\mathrm{e}^{-i k \xi}}{2 \pi i k}+\mathcal{O}\left(\frac{1}{k^{2}}\right)$, and hence, (3.1) reads

$$
\tilde{S}_{N}^{\sigma}[f](x)=[f](\xi) \sum_{k=1}^{N} \frac{\sigma\left(\frac{k}{N}\right)}{k} \cos k(x-\xi)+\mathcal{O}\left(\frac{\log N}{N}\right) .
$$

This leads us to seek admissible concentration factors, $\sigma\left(\frac{k}{N}\right)$, consult [6] and [7], satisfying

$$
\sum_{k=1}^{N} \frac{\sigma\left(\frac{k}{N}\right)}{k} \cos k(x-\xi) \rightarrow\left\{\begin{array}{l}
1, \text { if } x=\xi \\
0 \text { else }
\end{array}\right.
$$

so that the following concentration property holds

$$
\tilde{S}_{N}^{\sigma}[f](x) \rightarrow \begin{cases}{[f](\xi),} & \text { if } x=\xi \\ 0 & \text { otherwise. }\end{cases}
$$

We quote the main result in [7].

Corollary 3.1. The concentration property (3.3) holds for

$$
\int_{0}^{1} \frac{\sigma(s)}{s} \mathrm{~d} s=1, \quad \frac{\sigma(s)}{s} \in C^{2}[0,1] .
$$


In [6] the vanishing rate of $\tilde{S}_{N}^{\sigma}[f](x)$ was shown to have an upper bound of $\mathcal{O}\left(\frac{\log N}{N}\right)$. Different families of admissible concentration factors are discussed in [6] and [7]. Here we consider the following three examples for $\sigma(s), s=\frac{|k|}{N}$ :

1. Polynomial factors, e.g., $[2,13,3,8,11,6]$ :

$$
\sigma^{\mathrm{pol}}(s)=p s^{p}
$$

2. Trigonometric factors $[4,5]$ :

$$
\sigma^{\sin }(s)=\frac{\sin \alpha s}{S i(\alpha)}, \quad S i(\alpha)=\int_{0}^{\alpha} \frac{\sin \eta}{\eta} \mathrm{d} \eta .
$$

3. Exponential factors [7]:

$$
\sigma^{\exp }(s)=\text { Const } \cdot \mathrm{e}^{\frac{1}{\gamma s(s-1)}}, \quad \text { Const }=\int \exp \left(\frac{-1}{\gamma \eta(\eta-1)}\right) \mathrm{d} \eta,
$$

normalized so that

$$
\int_{s=0}^{1} \frac{\sigma^{\exp }(s)}{s} \mathrm{~d} s=1
$$

Now suppose that instead of the continuous Fourier coefficients, $\hat{f}_{k}$, we are given the discrete grid values, $f\left(x_{j}\right)$, defined at the $2 N+1$ equidistant points, $x_{j}:=-\pi+j \Delta x$, with $\Delta x:=\frac{2 \pi}{2 N+1}$. A jump discontinuity at $x=\xi$ is identified by its enclosed grid cell, $x_{j} \leq \xi \leq x_{j+1}$, and is characterized by the asymptotic statement

$$
f\left(x_{j+1}\right)-f\left(x_{j}\right)= \begin{cases}{[f](\xi)+\mathcal{O}(\Delta x)} & \text { for } j=j_{\xi}: \xi \in\left[x_{j}, x_{j+1}\right], \\ \mathcal{O}(\Delta x) & \text { for other } j^{\prime} s \neq j_{\xi} .\end{cases}
$$

As expressed in (3.8), every grid value experiences a jump discontinuity. The jumps that are of order $\mathcal{O}(\Delta x)$ are acceptable, but the $\mathcal{O}(1)$ jumps indicate a jump discontinuity in the underlying function $f(x)$. It is plausible then to detect the edges of $f(x)$ with (3.8) by simply comparing neighboring points. In fact, as will be shown below, there are some cases where the edge detection method based on the continuous Fourier coefficients, (3.1), has an equivalent interpretation as the application of either divided differences or differentiation in physical space. As an alternative, we propose an edge detection method based on the discrete Fourier coefficients which cannot be realized as a difference operator in physical space, and we demonstrate a sharper localization at the edges, due to the faster convergence rate in the smooth part of $f(x)$.

As an analog to (3.1), we consider the corresponding general concentration sum

$$
\tilde{T}_{N}^{\tau}[f](x):=\pi i \sum_{k=-N}^{N} \operatorname{sgn}(k) \tau\left(\frac{|k|}{N+\frac{1}{2}}\right) \tilde{f}_{k} \mathrm{e}^{i k x},
$$

where $\tilde{f}_{k}$ are computed in (2.2). As in the continuous case, we seek admissible concentration factors, $\tau\left(\frac{|k|}{N+\frac{1}{2}}\right)$, such that the concentration property holds:

$$
\tilde{T}_{N}^{\tau}[f](x) \rightarrow \begin{cases}{[f](\xi),} & \text { if } x=\xi \\ 0 & \text { otherwise. }\end{cases}
$$


In $[6$, Th. 4.1] it was shown that the discrete concentration factors correspond to the continuous concentration factors as

$$
\tau\left(\frac{|k| \Delta x}{\pi}\right)=\sigma\left(\frac{|k| \Delta x}{\pi}\right) \frac{\sin \frac{|k| \Delta x}{2}}{\frac{|k| \Delta x}{2}}, \quad \Delta x=\frac{\pi}{N+\frac{1}{2}}
$$

To summarize the derivation of this relationship, we begin by rearranging (3.9) as

$$
\tilde{T}_{N}^{\tau}[f](x)=\Delta x \sum_{k=1}^{N} \tau\left(\frac{k \Delta x}{\pi}\right)\left(\cos k x \sum_{j=0}^{2 N} f\left(x_{j}\right) \sin k x_{j}-\sin k x \sum_{j=0}^{2 N} f\left(x_{j}\right) \cos k x_{j}\right) .
$$

Substituting the expressions

$$
\sin k x_{j}=-\frac{\cos k x_{j+\frac{1}{2}}-\cos k x_{j-\frac{1}{2}}}{2 \sin \frac{k \Delta x}{2}} \quad \text { and } \quad \cos k x_{j}=\frac{\sin k x_{j+\frac{1}{2}}-\sin k x_{j-\frac{1}{2}}}{2 \sin \frac{k \Delta x}{2}}
$$

into (3.12) and summing by parts leads to

$$
\begin{aligned}
& \tilde{T}_{N}^{\tau}[f](x)=\sum_{k=1}^{N} \frac{\Delta x}{2 \sin \frac{k \Delta x}{2}} \tau\left(\frac{k \Delta x}{\pi}\right)\left(\sin k x \sum_{j=0}^{2 N}\left(f\left(x_{j+1}\right)-f\left(x_{j}\right)\right) \sin k x_{j+\frac{1}{2}}+\right. \\
& \left.+\cos k x \sum_{j=0}^{2 N}\left(f\left(x_{j+1}\right)-f\left(x_{j}\right)\right) \cos k x_{j+\frac{1}{2}}\right) .
\end{aligned}
$$

To evaluate the sums on the right, we first identify the discontinuous cell (and in general, finitely many like it), by its midpoint, $\xi_{j+\frac{1}{2}}:=x_{j_{\xi}+\frac{1}{2}}$. We then find that

$$
\sum_{j=0}^{2 N}\left(f\left(x_{j+1}\right)-f\left(x_{j}\right)\right) \sin k x_{j+\frac{1}{2}}=([f](\xi)+\mathcal{O}(\Delta x)) \sin k \xi_{j+\frac{1}{2}}+\mathcal{O}\left(\frac{\Delta x}{\sin \frac{k \Delta x}{2}}\right) .
$$

Here we argue as follows. The first term on the right of (3.15) is the contribution of the single jump at $j=j_{\xi}$. For the remaining terms, $j \neq j_{\xi}$, we use (3.13) to sum by parts once more, accumulating $2 N-2 \sim \frac{1}{\Delta x}$ terms of order $f\left(x_{j+1}\right)-2 f\left(x_{j}\right)+f\left(x_{j-1}\right) \sim \mathcal{O}(\Delta x)^{2}$ and two (or finitely many) 'boundary terms' of order $f\left(x_{j+1}\right)-f\left(x_{j}\right) \sim \mathcal{O}(\Delta x)$. These amount to the second term on the right of (3.15). The same argument yields

$$
\sum_{j=0}^{2 N}\left(f\left(x_{j+1}\right)-f\left(x_{j}\right)\right) \cos k x_{j+\frac{1}{2}}=([f](\xi)+\mathcal{O}(\Delta x)) \cos k \xi_{j+\frac{1}{2}}+\mathcal{O}\left(\frac{\Delta x}{\sin \frac{k \Delta x}{2}}\right) .
$$

Inserting (3.15) and (3.16) back into (3.14) we end up with

$$
\begin{aligned}
\tilde{T}_{N}^{\tau}[f](x)=\sum_{k=1}^{N} \frac{\Delta x}{2 \sin \frac{k \Delta x}{2}} \tau\left(\frac{k \Delta x}{\pi}\right)\left([f](\xi) \cos k x \cos k \xi_{j+\frac{1}{2}}+[f](\xi) \sin k x \sin k \xi_{j+\frac{1}{2}}\right) \\
\quad+\mathcal{O}(1) \cdot \Delta x \sum_{k=1}^{N} \frac{\Delta x}{\sin \frac{k \Delta x}{2}} \tau\left(\frac{k \Delta x}{\pi}\right)+\mathcal{O}(1) \cdot \sum_{k=1}^{N}\left(\frac{\Delta x}{\left.\sin \frac{k \Delta x}{2}\right)^{2} \tau\left(\frac{k \Delta x}{\pi}\right) .}\right.
\end{aligned}
$$


The second expression on the right is of order $\sim \Delta x|\log \Delta x|$, since $\tau(\cdot)$ is bounded. The third term is also $\mathcal{O}(\Delta x|\log \Delta x|)$, after summation by parts which takes into account the $C^{1}$ bound of $\tau(s):=\sigma(s) \times \sin \frac{\pi s}{2} / \frac{\pi s}{2}$, and the fact that $\tau\left(\frac{1}{N}\right) \sim \sigma\left(\frac{1}{N}\right) \leq \mathcal{O}(1 / N)$. We conclude

$$
\tilde{T}_{N}^{\tau}[f](x)=[f](\xi) \sum_{k=1}^{N} \frac{\sigma\left(\frac{k \Delta x}{\pi}\right)}{k} \cos k\left(x-\xi_{j+\frac{1}{2}}\right)+\mathcal{O}(\Delta x|\log \Delta x|) .
$$

Observe that as $\Delta x \rightarrow 0$, the discrete concentration sum, $\tilde{T}_{N}^{\tau}[f](x)$, as written in (3.18), agrees with the continuous concentration sum, (3.2). The comparison of (3.2) and (3.18) implies the relationship of the discrete and continuous concentration factors given by (3.11).

The three examples of admissible continuous concentration factors mentioned above correspond to the following discrete concentration factors $\tau=\tau\left(s_{k}\right)$, where $s_{k}=|k| \Delta x / \pi$.

1. Polynomial factors (with $p=1$ ).

$$
\tau^{\mathrm{pol}}\left(s_{k}\right)=s_{k} \frac{\sin \frac{\pi s_{k}}{2}}{\frac{\pi s_{k}}{2}}=\frac{2 \sin \frac{\pi s_{k}}{2}}{\pi} .
$$

2. Trigonometric factors:

$$
\tau^{\sin }\left(s_{k}\right)=\frac{\sin \alpha s_{k}}{\operatorname{Si}(\alpha)} \frac{\sin \frac{\pi s_{k}}{2}}{\frac{\pi s_{k}}{2}}
$$

3. Exponential factors:

$$
\tau^{\exp }\left(s_{k}\right)=\text { Const } \cdot \mathrm{e}^{\frac{1}{\gamma s_{k}\left(s_{k}-1\right)}} \frac{2 \sin \frac{\pi s_{k}}{2}}{\pi}, \quad \text { Const }=\int \exp \left(\frac{-1}{\gamma \eta(\eta-1)}\right) \mathrm{d} \eta,
$$

normalized so that

$$
\int_{s=0}^{1} \frac{\tau^{\exp }(s)}{s} \mathrm{~d} s=1
$$

What is the relationship between the edge detection formula (3.9) and the interpretation of edges in physical space (3.8)? More specifically, is there physical meaning for the edge detection formula? We begin by writing the physical space representation of (3.9) (consult (3.12)) as

$$
\tilde{T}_{N}^{\tau}[f](x):=-\Delta x \sum_{j=0}^{2 N} f\left(x_{j}\right) \sum_{k=1}^{N} \tau\left(\frac{k \Delta x}{\pi}\right) \sin k\left(x-x_{j}\right),
$$

and take the first order polynomial concentration factor (3.19) as our first example. Substituting $\tau^{\text {pol }}$ into (3.22) gives

$$
\tilde{T}_{N}^{\tau}[f](x)=-\frac{2 \Delta x}{\pi} \sum_{j=0}^{2 N} f\left(x_{j}\right) \sum_{k=1}^{N} \sin \frac{k \Delta x}{2} \sin k\left(x-x_{j}\right) .
$$

Utilizing the identity

$$
\cos k\left(x-x_{j+\frac{1}{2}}\right)-\cos k\left(x-x_{j-\frac{1}{2}}\right)=2 \sin \frac{k \Delta x}{2} \sin k\left(x-x_{j}\right),
$$


enables us to sum by parts (3.23), and noting that due to periodicity $\sum_{0}^{2 N}\left(f\left(x_{j+1}\right)-f\left(x_{j}\right)\right)=0$ we end up with

$$
\tilde{T}_{N}^{\tau}[f](x)=\Delta x \sum_{j=0}^{2 N}\left(f\left(x_{j+1}\right)-f\left(x_{j}\right)\right) D_{N}\left(x-x_{j+\frac{1}{2}}\right),
$$

where $D_{N}(y)$ is the usual Dirichlet sum, $D_{N}(y)=\left(1+2 \sum_{k=1}^{N} \cos k y\right) / 2 \pi$. Hence, (3.24) tells us that the discrete concentration kernel associated with the polynomial factor (3.19) amounts to interpolation of the local differences, $f\left(x_{j}\right)-f\left(x_{j+1}\right)$, at the intermediate grid points, $x_{j+\frac{1}{2}}$, implying that the discrete polynomial concentration kernel is nothing but comparing neighboring grid point data in physical space. Similarly, concentration kernels associated with the higher order polynomial factors, $\tau^{\mathrm{pol}}(s)=p s^{p} \times \sin \frac{\pi s}{2} / \frac{\pi s}{2}$ with odd $p$ 's, lead to $(p-1)$ th differentiation of the same interpolant of the differences.

Turning to our second example of trigonometric concentration factors, (3.20), the physical representation (3.22) with $\alpha=\pi$ becomes

$$
\tilde{T}_{N}^{\tau}[f](x)=-\frac{2}{S i(\pi)} \sum_{j=0}^{2 N} f\left(x_{j}\right) \sum_{k=1}^{N} \frac{\sin k \Delta x}{k} \sin \frac{k \Delta x}{2} \sin k\left(x-x_{j}\right) .
$$

Clearly, using the trigonometric factors also correspond to a form of interpolation, as is seen by comparing (3.25) and (3.23). However, in this case the interpolation is global, which is apparent in the additional summation term $\frac{\sin k \Delta x}{k}$. It is interesting to note that the trigonometric polynomial concentration factor (3.6) for the continuous case was originally derived in [1] in real space by looking at the local differences of the global partial sums. Specifically, the Gibbs' overshoot at the discontinuity was used to detect the locations of the discontinuities as

$$
\frac{S_{N}[f]\left(x+\frac{\pi}{N}\right)-S_{N}[f]\left(x-\frac{\pi}{N}\right)}{\frac{2}{\pi} \operatorname{Si}(\pi)} \rightarrow\left\{\begin{array}{lr}
{[f](\xi)} & \text { for } x=\xi \\
0 & \text { otherwise. }
\end{array}\right.
$$

The physical space interpretation of divided differences is immediately apparent from (3.26). The trigonometric factors (3.6) are readily deduced from expanding the partial sums and applying integration by parts to the Fourier coefficients.

Is it that all concentration factors translate into interpolation or differentiation in the physical space? In fact, the exponential concentration factor, $\tau^{\exp }$, has no such physical interpretation. Therefore the alternative approach of computing the discrete Fourier coefficients and detecting the edges from the pseudo-spectral sum (3.9) with $\tau=\tau^{\exp }$ yields a sharper localization of the edges of $f(x)$ than both $\tau^{\text {pol }}$ and $\tau^{\text {sin }}$, as the $\mathcal{O}(1)$ scale of the edges are better separated from the exponentially small values $\tau^{\exp }$ away from the edges of $f$ (consult [7, Sect. 2, Ex. 3]). We close by noting that although our discussion is limited to edge detection of functions with a single jump discontinuity, (3.9) applies to any piecewise smooth function.

\subsection{Numerical examples of the edge detection method}

To demonstrate the efficacy of (3.9), consider the examples:

$$
f_{a}(x):=\left\{\begin{array}{l}
\sin \left(\frac{1}{2}(x+\pi)\right), \quad x<0, \\
\sin \left(\frac{1}{2}(3 x-\pi)\right), x>0 .
\end{array} ; \quad f_{b}(x):=\left\{\begin{array}{l}
\cos \left(x-\frac{x}{2} \operatorname{sgn}\left(|x|-\frac{\pi}{2}\right)\right), \quad x<0, \\
\cos \left(\frac{5}{2} x+x \operatorname{sgn}\left(|x|-\frac{\pi}{2}\right)\right), x>0 .
\end{array}\right.\right.
$$



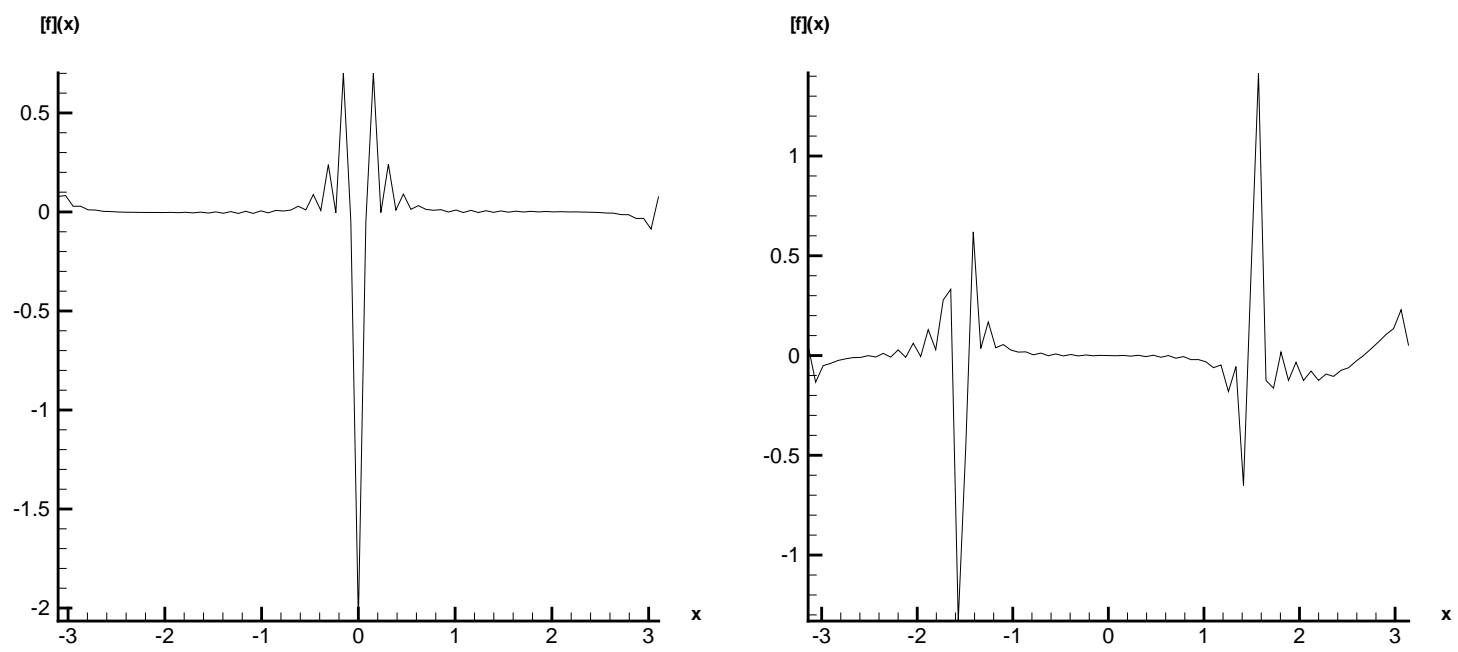

FIGURE 3.1. The edge detection method $\tilde{T}_{80}^{\tau}[f](x)$ with $\tau=\tau^{\text {exp }}$ to approximate (a) $\left[f_{a}\right](x)$ and $(\mathrm{b})\left[f_{b}\right](x)$. We use the parameters $\gamma=6$ yielding Const $\approx 3$ in $(3.21)$.
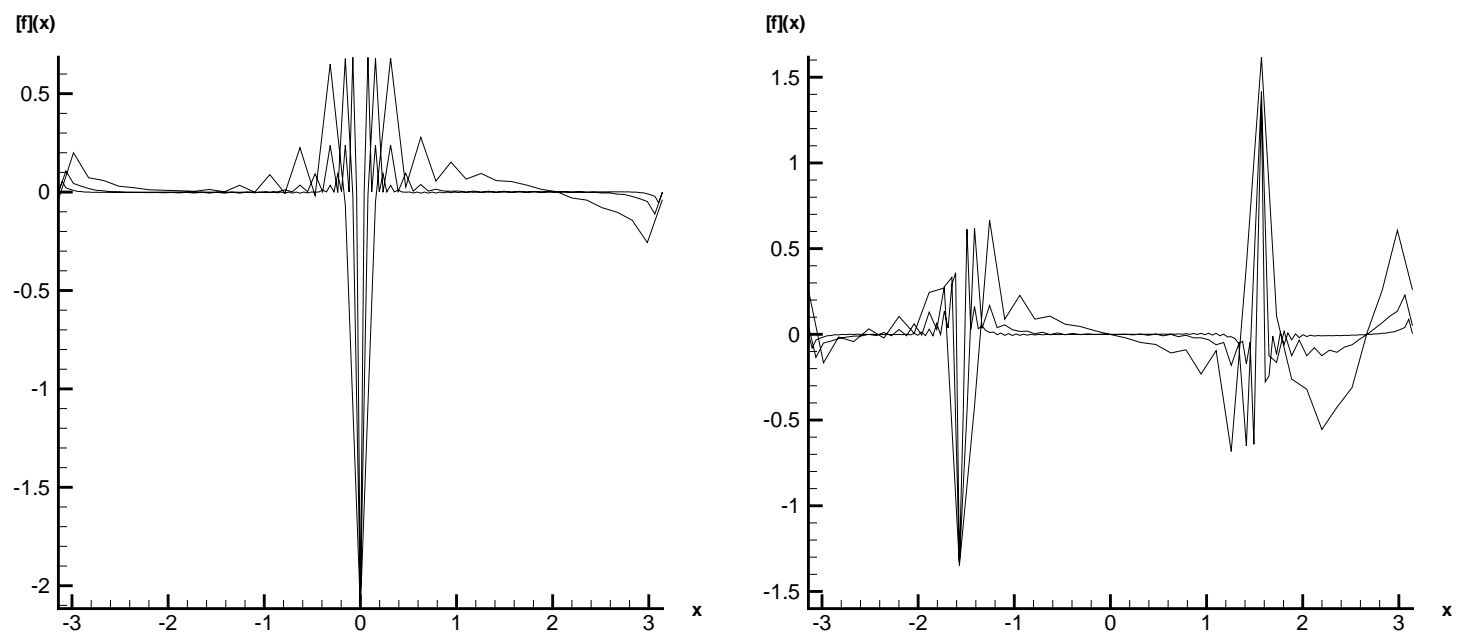

FigurE 3.2. The edge detection method $\tilde{T}_{N}^{\tau}[f](x)$ with $\tau=\tau^{\exp }$ with $\gamma=6$ used to approximate $(\mathrm{a})\left[f_{a}\right](x)$ and $(\mathrm{b})\left[f_{b}\right](x)$ with 40,80 , and 160 collocation points.

The corresponding jump functions are then

$$
\left[f_{a}\right](x):=\left\{\begin{array}{l}
-2, x=0, \\
0 \quad \text { else. }
\end{array} ; \quad\left[f_{b}\right](x):=\left\{\begin{array}{l} 
\pm \sqrt{2}, x= \pm \frac{\pi}{2} \\
0 \quad \text { else. }
\end{array}\right.\right.
$$

In Figure 3.1 we utilize the exponential concentration factor $\tau^{\exp }$ in (3.9) which provides a sharp localization of the jump discontinuities of $\left[f_{a}\right](x)$ and $\left[f_{b}\right](x)$. Furthermore, one should notice that the edge detection method specifically locates the $\epsilon$ neighborhoods in which the jump discontinuities occur, critical to the reconstruction method in Section 2. Figure 3.2 exhibits the rapid convergence of $\tilde{T}_{N}^{\tau}[f](x) \rightarrow 0$ away from the discontinuities of $f(x)$ using the exponential concentration factor. 


\subsection{Edge detection in two dimensions}

In Section 2.3, we used the jump discontinuities of $f(x, y)$ for the fixed intermediate grid points $\bar{x}$ and $\bar{y}$ to locate the smooth regions of $f(x, y)$ necessary for performing the localized reconstruction method (2.12). To determine the jump discontinuities of $f(x, y)$,

$$
\xi_{i}(\bar{y}), i=1, \cdots M_{x}(\bar{y}), \quad \eta_{j}(\bar{x}), j=1, \cdots M_{y}(\bar{x}),
$$

on the fixed grid point values $\bar{y}$ and $\bar{x}$, we employ (3.9) to obtain

$$
\begin{aligned}
& \tilde{T}_{N}^{\tau}[f](x(\bar{y}))=\pi i \sum_{l=-N}^{N} \sum_{k=-N}^{N} \operatorname{sgn}(k) \tau\left(\frac{|k| \Delta x}{\pi}\right) \tilde{f}_{k, l} \mathrm{e}^{i k x(\bar{y})+i l \bar{y}}, \\
& \tilde{T}_{N}^{\tau}[f](y(\bar{x}))=\pi i \sum_{l=-N}^{N} \sum_{k=-N}^{N} \operatorname{sgn}(l) \tau\left(\frac{|l| \Delta y}{\pi}\right) \tilde{f}_{k, l} \mathrm{e}^{i k \bar{x}+i l y(\bar{x})},
\end{aligned}
$$

where the discrete coefficients of $f(x, y)$ are computed by

$$
\tilde{f}_{k, l}=\frac{1}{(2 N+1)^{2}} \sum_{i=0}^{2 N} \sum_{j=0}^{2 N} f\left(x_{i}, y_{j}\right) \mathrm{e}^{-i k x_{i}-i l y_{j}} .
$$

The concentration property is then given as

$$
\begin{aligned}
& \tilde{T}_{N}^{\tau}[f](x(\bar{y})) \rightarrow \begin{cases}{[f]\left(\xi_{i}(\bar{y})\right),} & \text { if } x=\xi_{i}(\bar{y}), i=1, . . M_{x}(\bar{y}), \\
0 & \text { otherwise },\end{cases} \\
& \tilde{T}_{N}^{\tau}[f](y(\bar{x})) \rightarrow \begin{cases}{[f]\left(\eta_{j}(\bar{x})\right),} & \text { if } y=\eta_{j}(\bar{x}), j=1, . . M_{y}(\bar{x}), \\
0 & \text { otherwise. }\end{cases}
\end{aligned}
$$

As an example, we consider the function

$$
f(x, y)= \begin{cases}3 \cos \frac{x y}{\pi}-\sin \frac{x}{2}-\sin \frac{y}{2}, & \text { if } x^{2}+y^{2}<(0.7 \pi)^{2} \\ 0 & \text { otherwise }\end{cases}
$$

and apply (3.28) using $\tau=\tau^{\text {exp }}$ in each direction. Figure 3.3 shows the convergence to the jump discontinuities of $[f](x, y)$ occurring at $x^{2}+y^{2}=(0.7 \pi)^{2}$. While the neighborhoods of the discontinuities are indeed detected, the limitations of the "Cartesian" detection done dimension by dimension are clear. Resolution is lost when the edges are not orthogonal to the Cartesian grid.

\section{Enhancement of the CONCENTRATion MEthod}

While (3.9) and (3.28) locate the neighborhoods of the discontinuities, the enhancement method introduced in [7] improves the detection by actually 'pinpointing' the edges. More specifically, the results in (3.9) and (3.28) are enhanced by amplifying the separation of scales. This is necessary in the localized reconstruction method, (2.10) and (2.12), to determine the regions of smoothness and avoid unnecessary smoothing near the discontinuities. We begin with the results of [7] as applied directly to (3.9).

Let $\left\{\xi_{j}\right\}$ denote the location of the jump discontinuities, $j=1, \cdots, M$. Then (3.9) is amplified by

$$
\left(\tilde{T}_{N}^{\tau}[f](x)\right)^{q} \rightarrow \begin{cases}\left([f]\left(\xi_{j}\right)\right)^{q}, & \text { if } x=\xi_{j}, \\ \mathcal{O}\left(\frac{1}{N}\right)^{q}, & \text { if } x \neq \xi_{j} .\end{cases}
$$




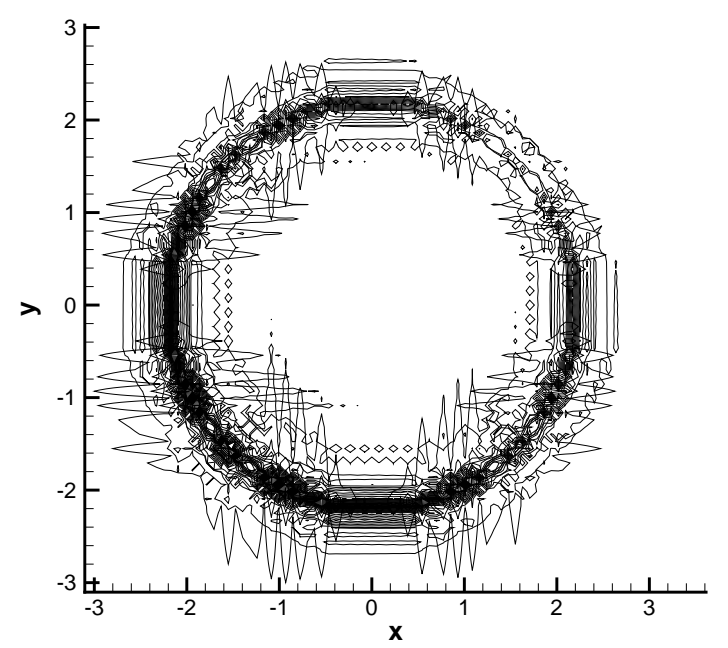

Figure 3.3. The edge detection methods $\tilde{T}_{80}^{\tau}[f](x(\bar{y}))$ and $\tilde{T}_{80}^{\tau}[f](y(\bar{x}))$ with $\tau=\tau^{\exp }$ applied to $(3.30)$.

A more pronounced separation of scales is easily accomplished by defining

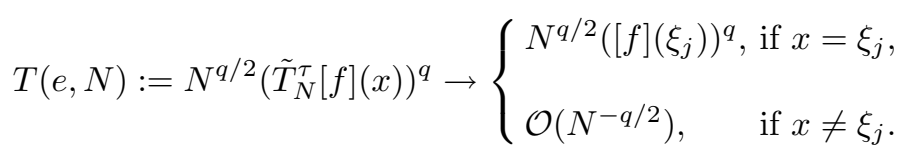

The enhanced edge detection method is then

$$
\tilde{T}_{N}^{\tau, e}[f](x)= \begin{cases}\tilde{T}_{N}^{\tau}[f](x), & \text { if }|T(e, N)|>J_{\text {crit }}, \\ 0, & \text { if }|T(e, N)|<J_{\text {crit }}\end{cases}
$$

where $J_{\text {crit }}$ is an $\mathcal{O}(1)$ global threshold parameter signifying the critical (minimal) amplitude necessary for the jump discontinuities to qualify as admissible. Any jumps with smaller amplitudes are ignored. In recognition that (3.9) and (3.28) actually detect the $\epsilon$-neighborhoods of the jump discontinuities, rather than the discontinuities themselves, we use the nonlinear enhancement (4.3) in a window of $\mathcal{O}(\epsilon(N))$ to pinpoint the exact locations. More specifically, we determine that the exact discontinuities are where the largest amplitude $|T(e, N)|>J_{\text {crit }}$ occur in each small neighborhood $\epsilon$ of the jump discontinuities. This is consistent with the fact that the jump discontinuities must be spaced at least $\mathcal{O}(\epsilon(N))$ away from each other for the function $f(x)$ to be properly resolved. The edges $x=\left\{\xi_{j}\right\}_{j=1}^{M}$ are the locations corresponding to the largest nonzero values of (4.3) in each neighborhood of the jump discontinuities. The obvious benefits of using the enhancement procedure (4.3) for $f_{a}(x)$ and $f_{b}(x)$ in $(3.27)$ are depicted in Figure 4.1 .

In two dimensions, pinpointing the edges is accomplished by the same technique of separating the scales (4.2), yielding

$$
\begin{gathered}
T_{x}:=N^{q / 2}\left(\tilde{T}_{N}^{\tau}[f](x(\bar{y}))\right)^{q} \rightarrow \begin{cases}N^{q / 2}\left([f]\left(\xi_{i}(\bar{y})\right)\right)^{q}, & \text { if } x=\xi_{i}(\bar{y}), i=1, \cdots, M_{x}(\bar{y}), \\
\mathcal{O}\left(N^{-q / 2}\right), & \text { if } x \neq \xi_{i}(\bar{y}), i=1, \cdots, M_{x}(\bar{y}),\end{cases} \\
T_{y}:=N^{q / 2}\left(\tilde{T}_{N}^{\tau}[f](y(\bar{x}))\right)^{q} \rightarrow \begin{cases}N^{q / 2}\left([f]\left(\eta_{j}(\bar{x})\right)\right)^{q}, & \text { if } y=\eta_{j}(\bar{x}), j=1, \cdots, M_{y}(\bar{x}), \\
\mathcal{O}\left(N^{-q / 2}\right), & \text { if } y \neq \eta_{j}(\bar{x}), j=1, \cdots, M_{y}(\bar{x}) .\end{cases}
\end{gathered}
$$



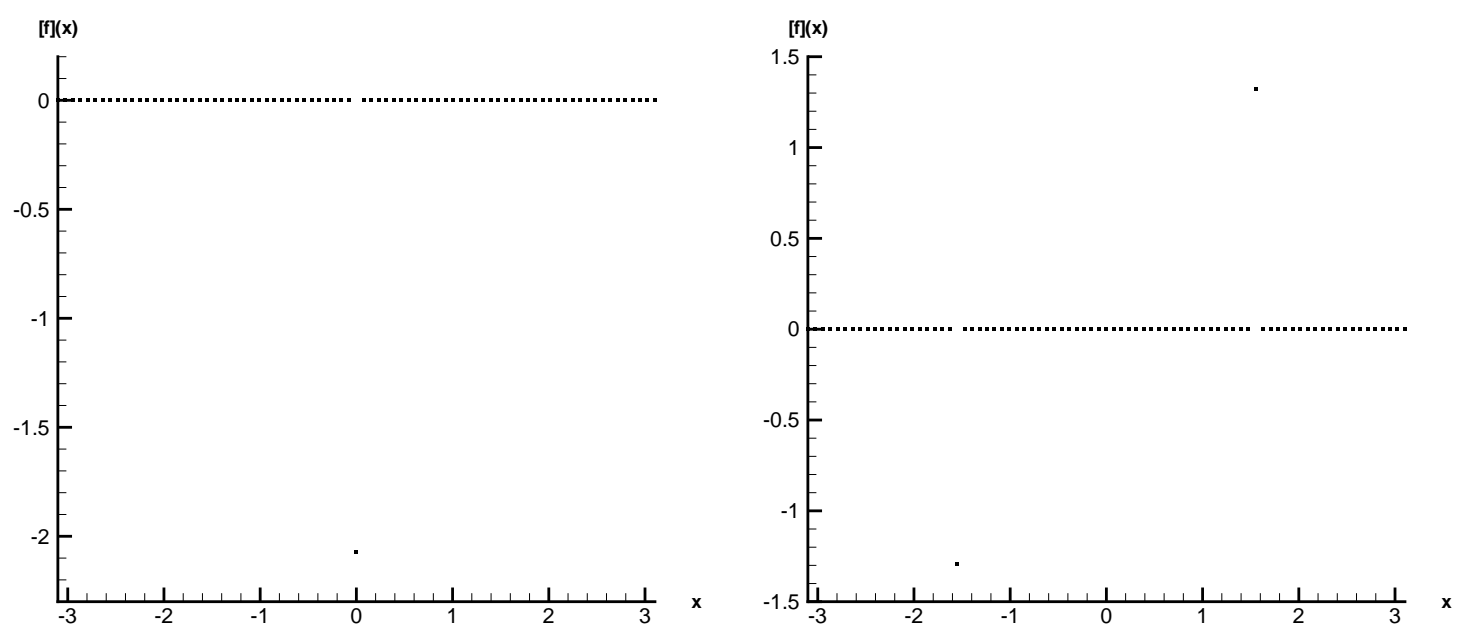

Figure 4.1. The edge enhancement procedure $\tilde{T}_{80}^{\tau, e}[f](x)$ with $J_{\text {crit }}=5$ is used to pinpoint the edges of $(\mathrm{a}) f_{a}(x)$ and $(\mathrm{b}) f_{b}(x)$ in $(3.27)$.

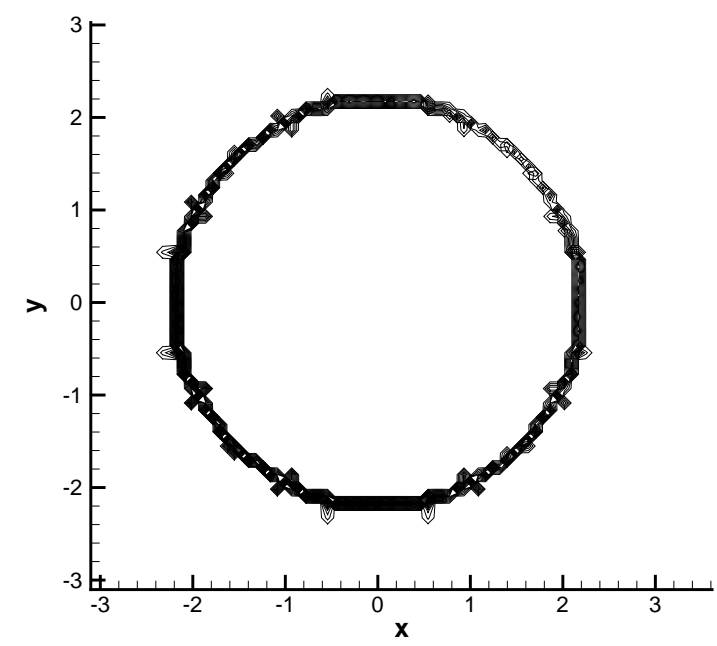

Figure 4.2. The nonlinear enhancement $\tilde{T}_{80}^{\tau, e}[f](x(\bar{y}))$ and $\tilde{T}_{80}^{\tau, e}[f](y(\bar{x}))$ applied to (3.30).

The enhancement procedure (4.3) yields

$$
\begin{aligned}
& \tilde{T}_{N}^{\tau, e}[f](x(\bar{y}))= \begin{cases}\tilde{T}_{N}^{\tau}[f](x(\bar{y})), & \text { if }\left|T_{x}\right|>J_{\text {crit }}, \\
0, & \text { if }\left|T_{x}\right|<J_{\text {crit }},\end{cases} \\
& \tilde{T}_{N}^{\tau, e}[f](y(\bar{x}))= \begin{cases}\tilde{T}_{N}^{\tau}[f](y(\bar{x})), & \text { if }\left|T_{y}\right|>J_{\text {crit }}, \\
0, & \text { if }\left|T_{y}\right|<J_{\text {crit }},\end{cases}
\end{aligned}
$$

where again we pinpoint the exact edges by determining the largest amplitudes of $\tilde{T}_{N}^{\tau, e}[f](x(\bar{y}))$ and $\tilde{T}_{N}^{\tau, e}[f](y(\bar{x}))$ in each neighborhood of the jump discontinuities. Figure 4.2 shows the enhancement of the edges as determined by (4.4) for (3.30). 


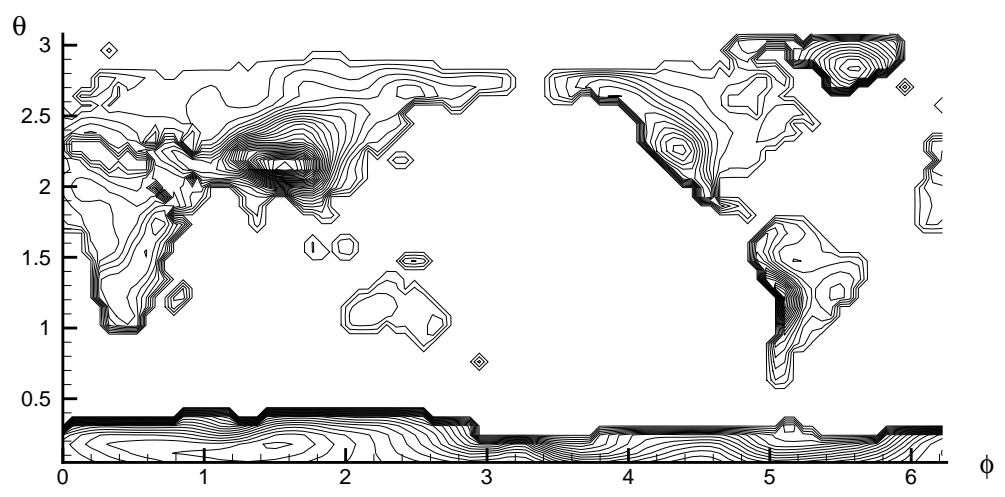

Figure 4.3. The earth's topography using 48 latitudinal grid points and 96 longitudinal grid points.
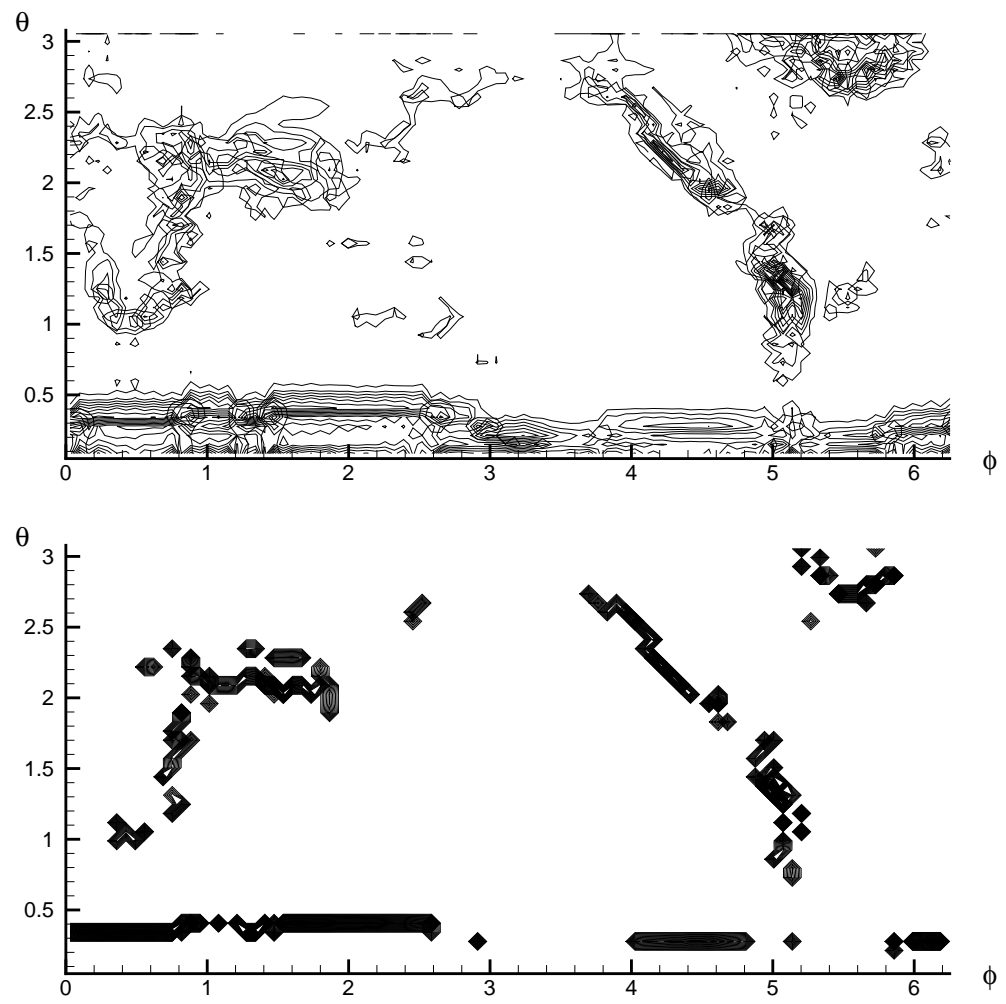

FigurE 4.4. The edges of the world's mountain ranges (a) before and (b) after nonlinear enhancement.

To close this section we consider a pictorial example of the applications of the edge detection and nonlinear enhancement procedures. Suppose we are given the grid point data of the function representing the earth's topography on a $48 \times 96$ grid as depicted in Figure 4.3. We wish to recover the locations of the mountain ranges with steep gradients. We note that the longitudinal data is given on the Legendre Gauss Lobatto grid points, and therefore use the edge detection for the Legendre case (consult [7]). The results shown in Figure 4.4 indicate that the nonlinear enhancement procedure is critical in identifying the exact edges of the mountain ranges. 


\section{NumERICAL RESUlTS OF THE LOCALIZED RECONSTRUCTION METHOD}

The results from Sections 2, 3 and 4 are combined to form a new localized reconstruction method, which accurately reconstructs a piecewise smooth function from its grid values. It can be easily described in the following steps:

1. The jump discontinuities of $f(x)$ are determined by the concentration kernels (3.9),

$$
\tilde{T}_{N}^{\tau}[f](x)=\pi i \sum_{k=-N}^{N} \operatorname{sgn}(k) \tau\left(\frac{|k| \Delta x}{\pi}\right) \tilde{f}_{k} \mathrm{e}^{i k x} \rightarrow \begin{cases}{[f](\xi),} & \text { if } x=\xi \\ 0 & \text { otherwise. }\end{cases}
$$

2. The location of these jumps is further 'pinpointed' by the nonlinear enhancement procedure (4.3),

$$
\tilde{T}_{N}^{\tau, e}[f](x)= \begin{cases}\tilde{T}_{N}^{\tau}[f](x), & \text { if }|T(e, N)|>J_{\text {crit }} \\ 0, & \text { if }|T(e, N)|<J_{\text {crit }}\end{cases}
$$

3. The function can now be reconstructed by the localized approximation (2.10), where in each region of smoothness $(w-\theta, w+\theta)$, dictated by the distance to the nearest jump $\theta=\max \left\{\min \left(\theta_{\text {left }}, \theta_{\text {right }}\right), \epsilon\right\}$, we set

$$
I_{N}^{\Psi}[f](w)=\frac{\Delta x}{2 \pi \theta} \sum_{\left|x_{j}-w\right|<\theta} f\left(x_{j}\right) \rho\left(\frac{w-x_{j}}{\theta}\right) \frac{\sin \left(p+\frac{1}{2}\right)\left(\frac{w-x_{j}}{\theta}\right)}{\sin \frac{1}{2}\left(\frac{w-x_{j}}{\theta}\right)} .
$$

In our examples we use the $C^{\infty}$ cut-off function

$$
\rho(\zeta)=\rho_{\alpha}(\zeta)= \begin{cases}\mathrm{e}^{\frac{\alpha \zeta^{2}}{\zeta^{2}-1}}, & \text { if }|\zeta|<1 \\ 0 & \text { otherwise }\end{cases}
$$

with $\alpha=10, p=N^{\beta}, \beta=0.8$, and $\epsilon \approx 5 \Delta x$.

In our experiments, $\beta \geq 0.7$ consistently yielded more desirable results than $\beta<0.7$, although no optimization attempt was made. The optimal choice for $\beta$ seems to correspond with both the function and the number of grid points in the approximation (the question of optimality was recently answered in [12], in terms of the adaptive choice, $p \sim \theta N)$.

We note that the pseudo-spectral coefficients are only needed in the first step, since the actual reconstruction is performed in physical space, yielding a cost-effective approximation. Spectral accuracy is obtained at the intermediate grid points outside the small neighborhoods of $y=\left\{\xi_{k}\right\}_{k=1}^{M}$.

Consider the same previous examples

$$
f_{a}(x):=\left\{\begin{array}{l}
\sin \left(\frac{1}{2}(x+\pi)\right), \quad x<0, \\
\sin \left(\frac{1}{2}(3 x-\pi)\right), x>0 .
\end{array} ; \quad f_{b}(x):=\left\{\begin{array}{l}
\cos \left(x-\frac{x}{2} \operatorname{sgn}\left(|x|-\frac{\pi}{2}\right)\right), \quad x<0, \\
\cos \left(\frac{5}{2} x+x \operatorname{sgn}\left(|x|-\frac{\pi}{2}\right)\right), x>0 .
\end{array}\right.\right.
$$

Figures 5.1 and 5.2 compare the Fourier sum approximation $I_{N}[f](x)$ to the new localized approximation $I_{N}^{\Psi}[f](x)$. The convergence rates are depicted in Figure 5.3. The logarithmic scale of the errors in Figure 5.3 indicates that the accuracy at intermediate grid point values $y=x_{j+\frac{1}{2}}$ away from the discontinuities is indeed spectral. Furthermore, the localized approximation yields much higher resolution than an approximation containing a standard exponential filter, as is evident in Figure 5.4.

For the two dimensional case, the same three step procedure is used: The neighborhoods of the jump discontinuities are detected via (3.28), followed by the nonlinear enhancement (4.4) to pinpoint the edges, and 

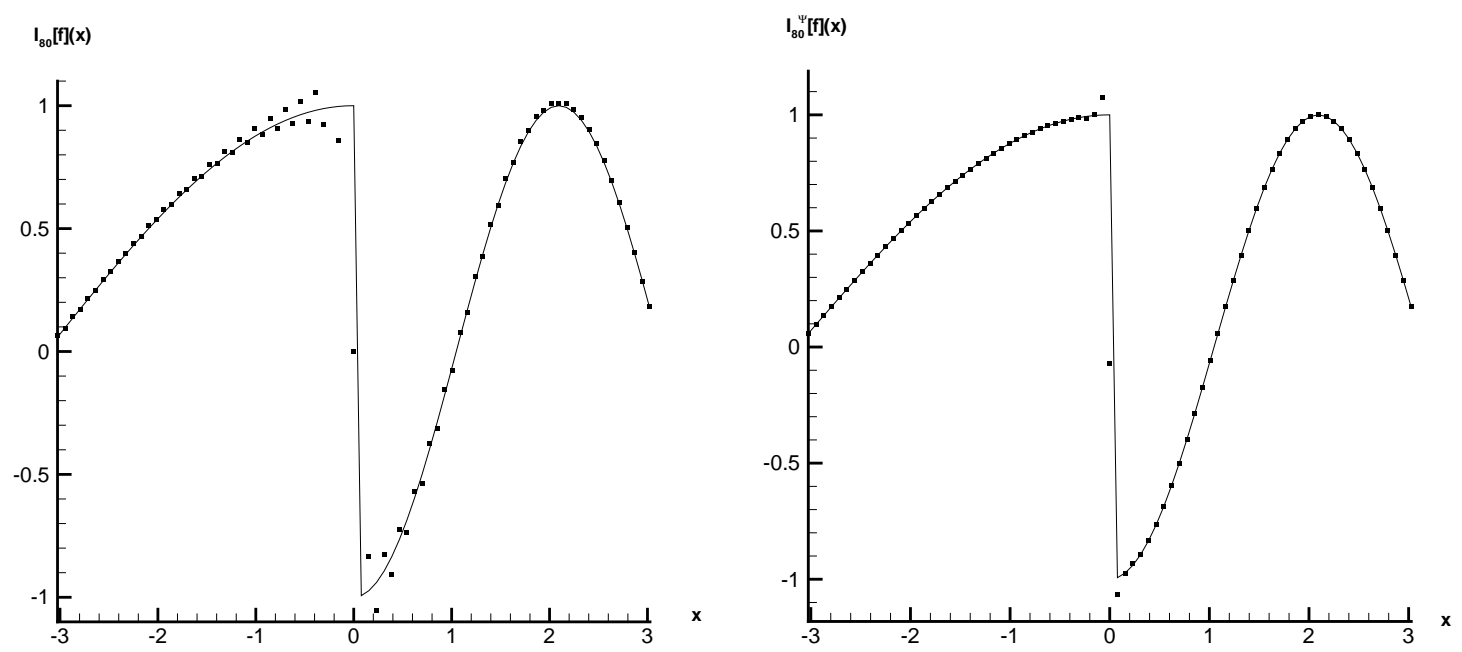

Figure 5.1. Approximation to $f_{a}(x)$ using the approximations (a) $I_{80}\left[f_{a}\right](x)$ and (b) $I_{80}^{\Psi}\left[f_{a}\right](x)$.
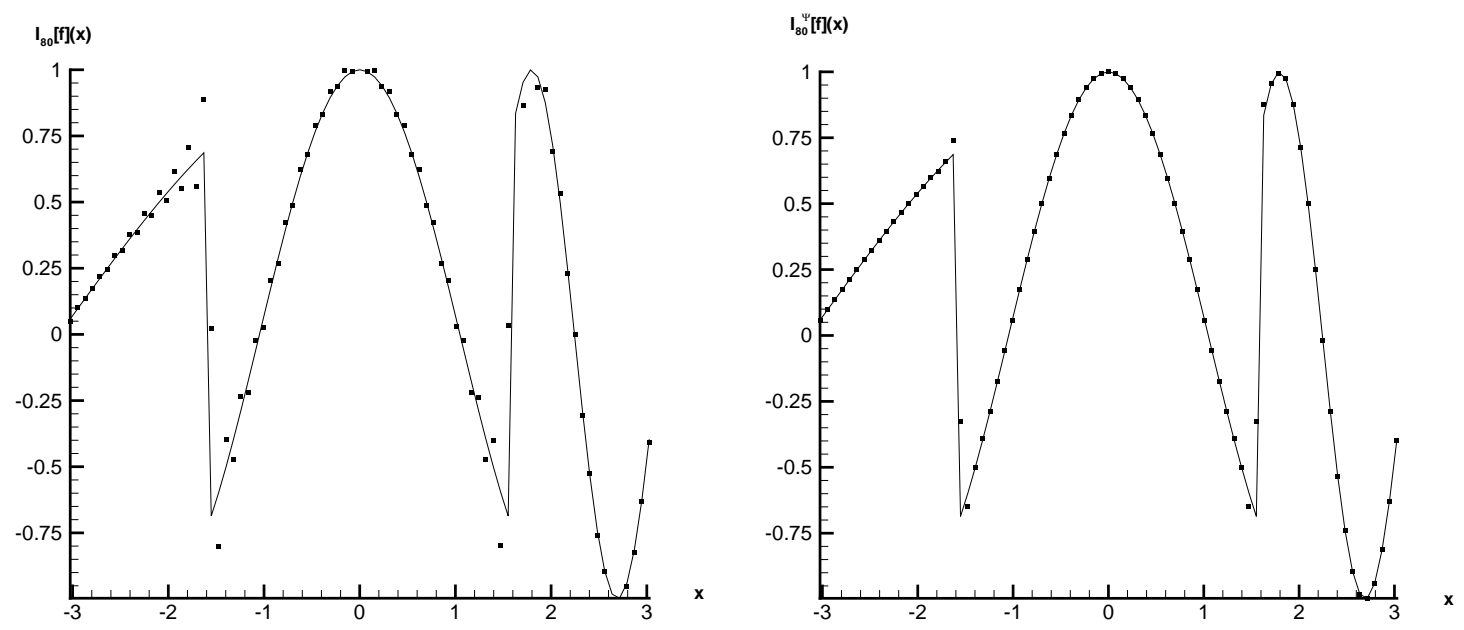

FiguRE 5.2. Approximation to $f_{b}(x)$ using the approximations (a) $I_{80}\left[f_{b}\right](x)$ and (b) $I_{80}^{\Psi}\left[f_{b}\right](x)$.

finally the localized reconstruction described in (2.12) is performed. We refer to the previous example,

$$
f(x, y)= \begin{cases}3 \cos \frac{x y}{\pi}-\sin \frac{x}{2}-\sin \frac{y}{2}, & \text { if } x^{2}+y^{2}<(0.7 \pi)^{2} \\ 0 & \text { otherwise }\end{cases}
$$

Figure 5.5 shows the contour plot and a one-dimensional cross section of $f(x, y)$. Figures 5.6 and 5.7 compare the solution obtained from the two-dimensional Fourier sum and the localized approximation (2.12) for which the discontinuities were determined by (4.4). Figure 5.8 shows the solution for $N=40$ and $N=160$ respectively. Table 1 depicts the errors for the localized approximation, $I_{N}^{\Psi^{\theta, p}}[f](x, y)$, with $\rho_{\alpha}, \alpha=10$ and $p=N^{\beta}, \beta=0.8$, at several grid points $(x, y)$, confirming that the intermediate grid point values away from the discontinuities exhibit spectral convergence. 

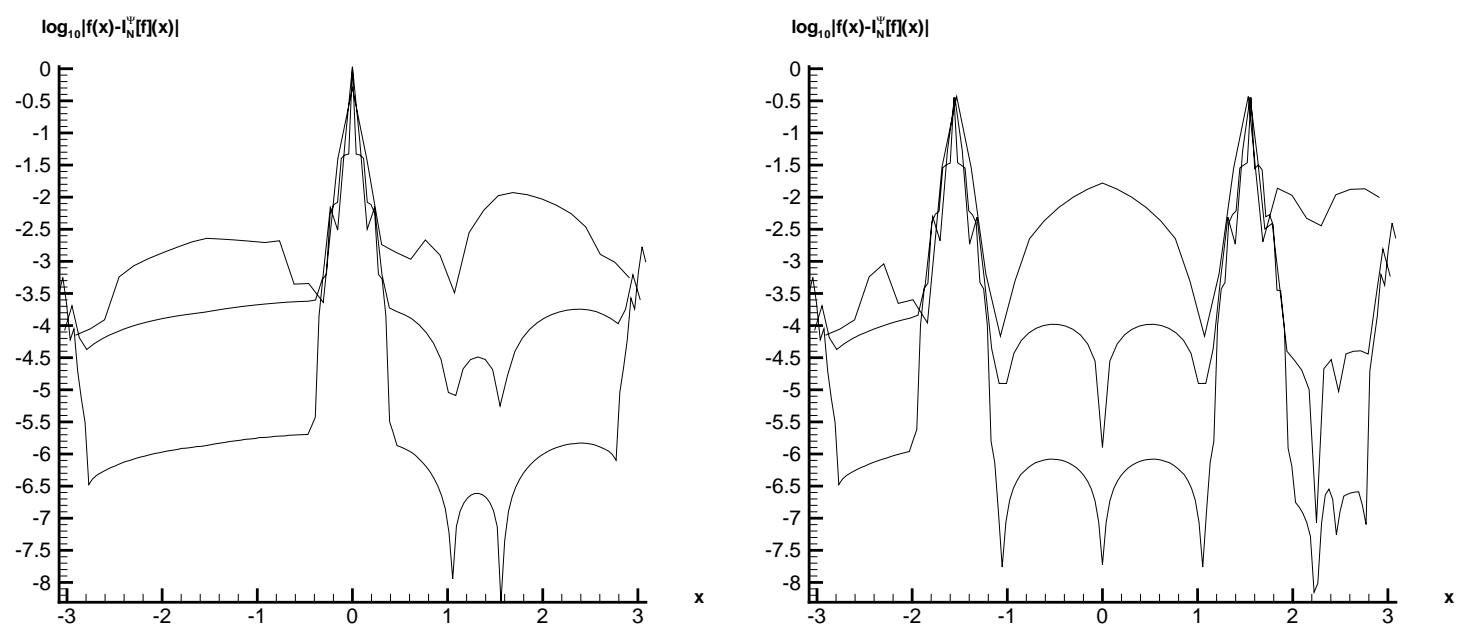

FiguRE 5.3. Logarithmic scale errors for (a) $I_{N}^{\Psi}\left[f_{a}\right](x)$ and (b) $I_{N}^{\Psi}\left[f_{b}\right](x)$ with $N=40,80,160$.
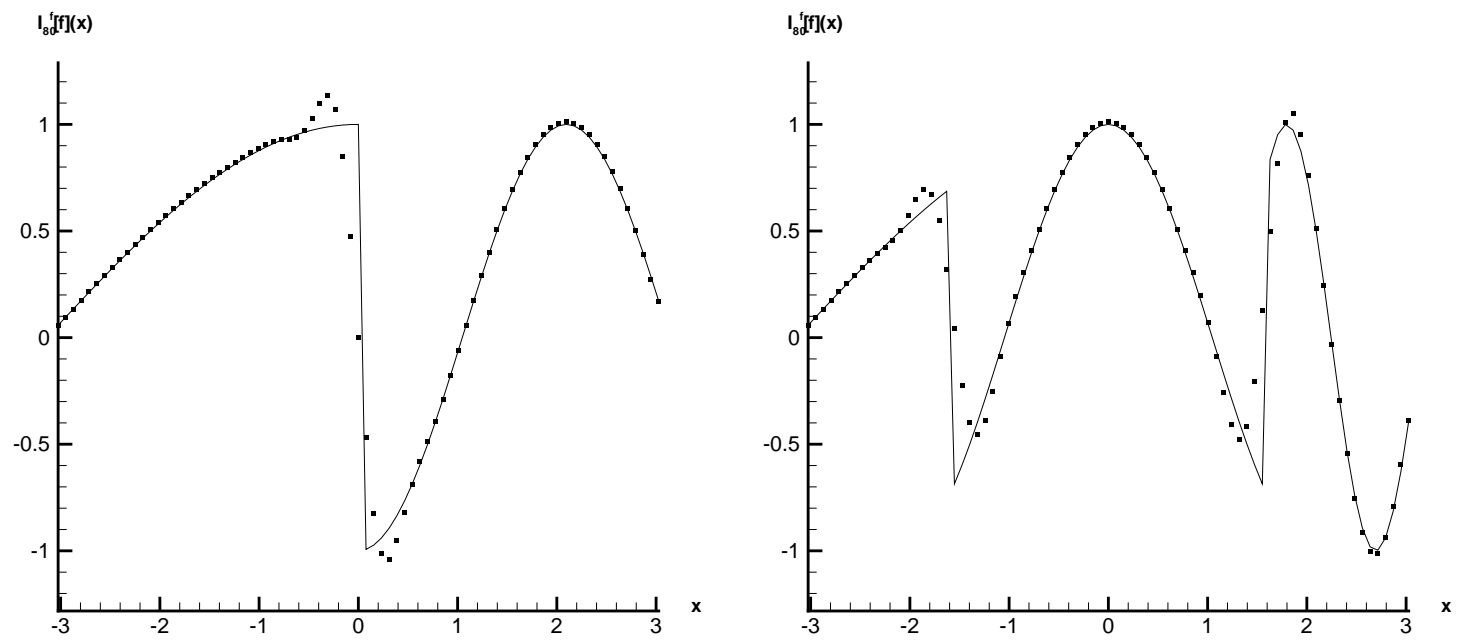

Figure 5.4. Approximations of (a) $f_{a}(x)$ and (b) $f_{b}(x)$ using a standard exponential filters with $N=80$ grid points.

TABLE 1. Error for the localized reconstruction method $I_{N}^{\Psi}[f](x, y)$.

\begin{tabular}{||c|c|c|l|l||}
\hline$N$ & $(x, y)=(0,0)$ & $(x, y)=\left(-\frac{\pi}{2},-\frac{\pi}{4}\right)$ & $(x, y)=\left(\frac{\pi}{2}, 0\right)$ & $(x, y)=\left(-\frac{\pi}{4},-\frac{\pi}{2}\right)$ \\
\hline 40 & $1.0 \mathrm{E}-02$ & $5.4 \mathrm{E}-03$ & $9.8 \mathrm{E}-03$ & $5.4 \mathrm{E}-03$ \\
80 & $1.5 \mathrm{E}-03$ & $1.9 \mathrm{E}-03$ & $1.1 \mathrm{E}-03$ & $1.9 \mathrm{E}-03$ \\
160 & $9.5 \mathrm{E}-06$ & $8.6 \mathrm{E}-06$ & $5.7 \mathrm{E}-06$ & $8.6 \mathrm{E}-06$ \\
\hline
\end{tabular}

To close this section, we return to the example of the earth's topography. Figure 5.9 compares the regular spectral approximation based on the pseudo-spectral coefficients (Fourier in the longitudinal direction and Legendre in the latitudinal direction) to the new localized reconstruction method. Here we adapted the physical 

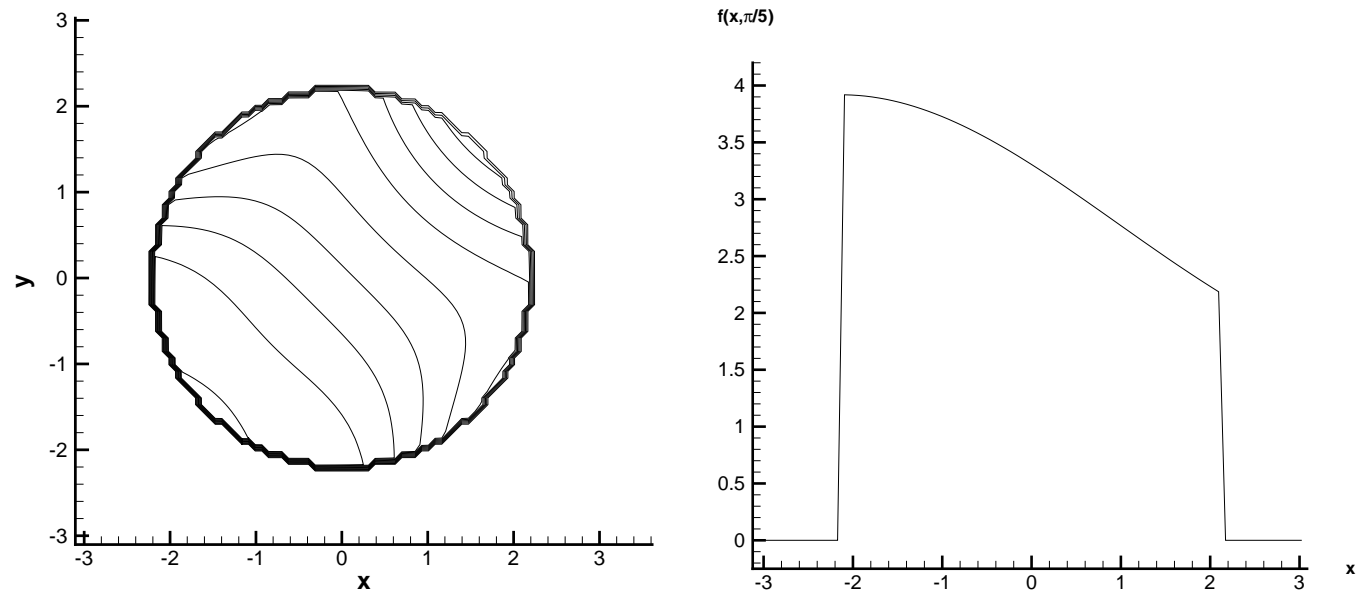

FiguRE 5.5. (a) contour plot of example (5.1) and (b) cross section at $y=0$.
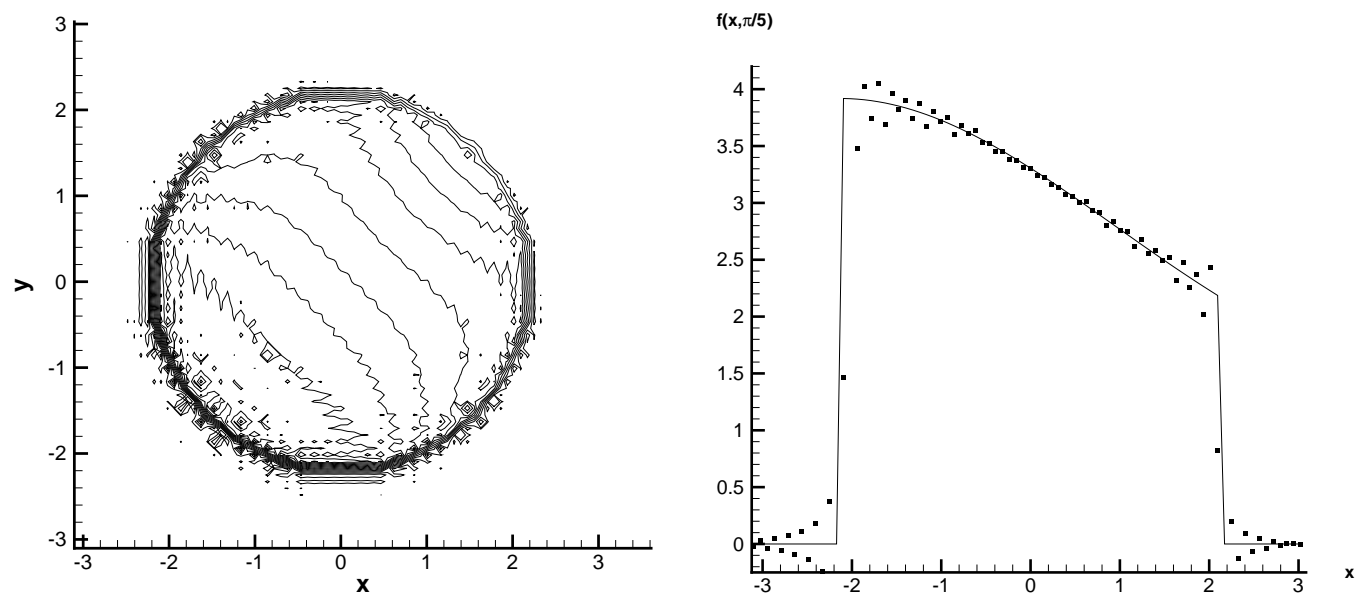

Figure 5.6. The Fourier sum approximation $I_{80}[f](x, y)$ to $(5.1)$.

space Legendre reconstruction method [10] in the latitudinal direction to include the edge detection and enhancement procedures from [7]. The Fourier localized reconstruction method discussed above was used in the longitudinal direction.

We stress that without the successful edge detection exhibited in Figure 4.4, the reconstruction would have resulted in either the occurrence of Gibbs' oscillations or the loss of amplitude in the mountains, both of which are highly undesirable in climatology modeling. It is evident that although Figure 5.9 removes the oscillations while maintaining the finer features of the earth's topography, there is definitely still work to be done. A case in point, the reconstruction should be performed in two dimensions directly instead of dimension by dimension.

\section{CONCLUDING REMARKS}

The new localized reconstruction method is a cost efficient way to reconstruct piecewise smooth functions in one and two dimensions. Although other reconstruction methods (e.g. [9] and references therein) yield exponential accuracy up to the jump discontinuities, the cost of the approximations are significantly higher due to the computational cost of the pseudo-spectral approximations. Furthermore, our edge detection and 

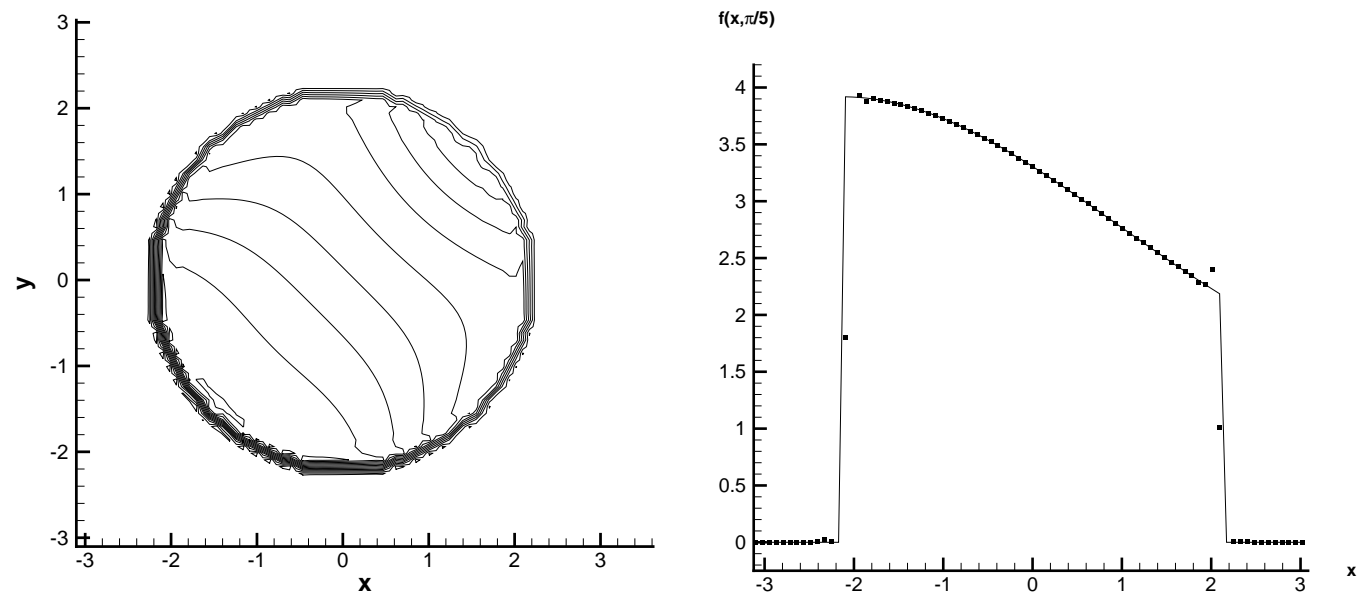

FIGURE 5.7. The localized approximation $I_{80}^{\Psi}[f](x, y)$ to $(5.1)$.
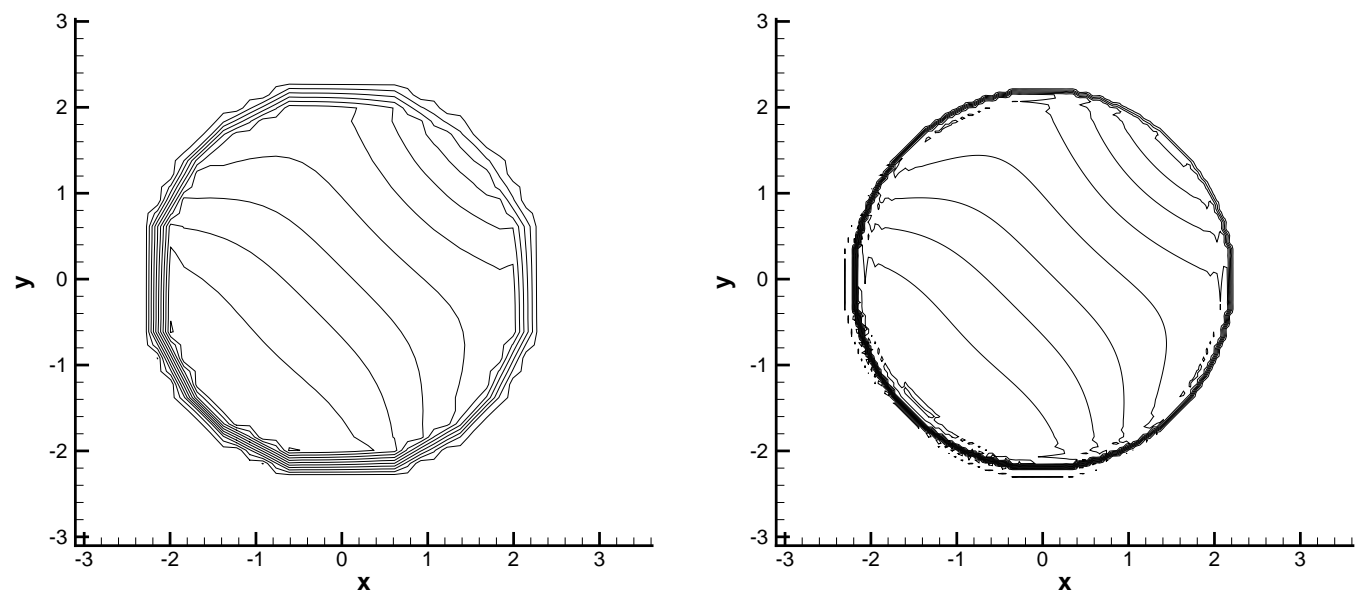

Figure 5.8. The localized reconstruction (a) $I_{40}^{\Psi}[f](x, y)$ and (b) $I_{160}^{\Psi}[f](x, y)$ to $(5.1)$.

enhancement methods are applicable to all reconstruction methods, including those cited in [9], potentially yielding greater accuracy and higher resolution. As it stands, the new localized reconstruction method discussed here provides an accurate approximation to intermediate grid point values, and is particularly effective away from the jump discontinuities.

Future considerations for the localized reconstruction method will include:

- Optimization of the post-processing parameters $\alpha$ and $p$ in the localizing regularization kernel (2.4), $\Psi^{\theta, p}(\zeta)=\rho_{\alpha}(\zeta) D_{p}(\zeta)$. As our examples indicate, accuracy is dependent on the choice of these parameters. Larger choices for $\alpha$ implies heavier filtering ( $\alpha=10$ being relatively large), while smaller choices of $\beta$ for $p=N^{\beta}$ result in eliminating more high frequency modes. In particular, an optimal choice based on the adaptive parameterization, $p \sim \theta N$ and the normalization of $\rho_{\alpha}$ advocated in [12, Sect. 4], should be further tested.

- Initial grid point data with noise. Several test cases were tried when the initial data was the pseudospectral approximation $f_{N}\left(x_{j}\right)$ of $f(x)$. The results concurred, but more examination is required. Other types of contamination should also be considered. 

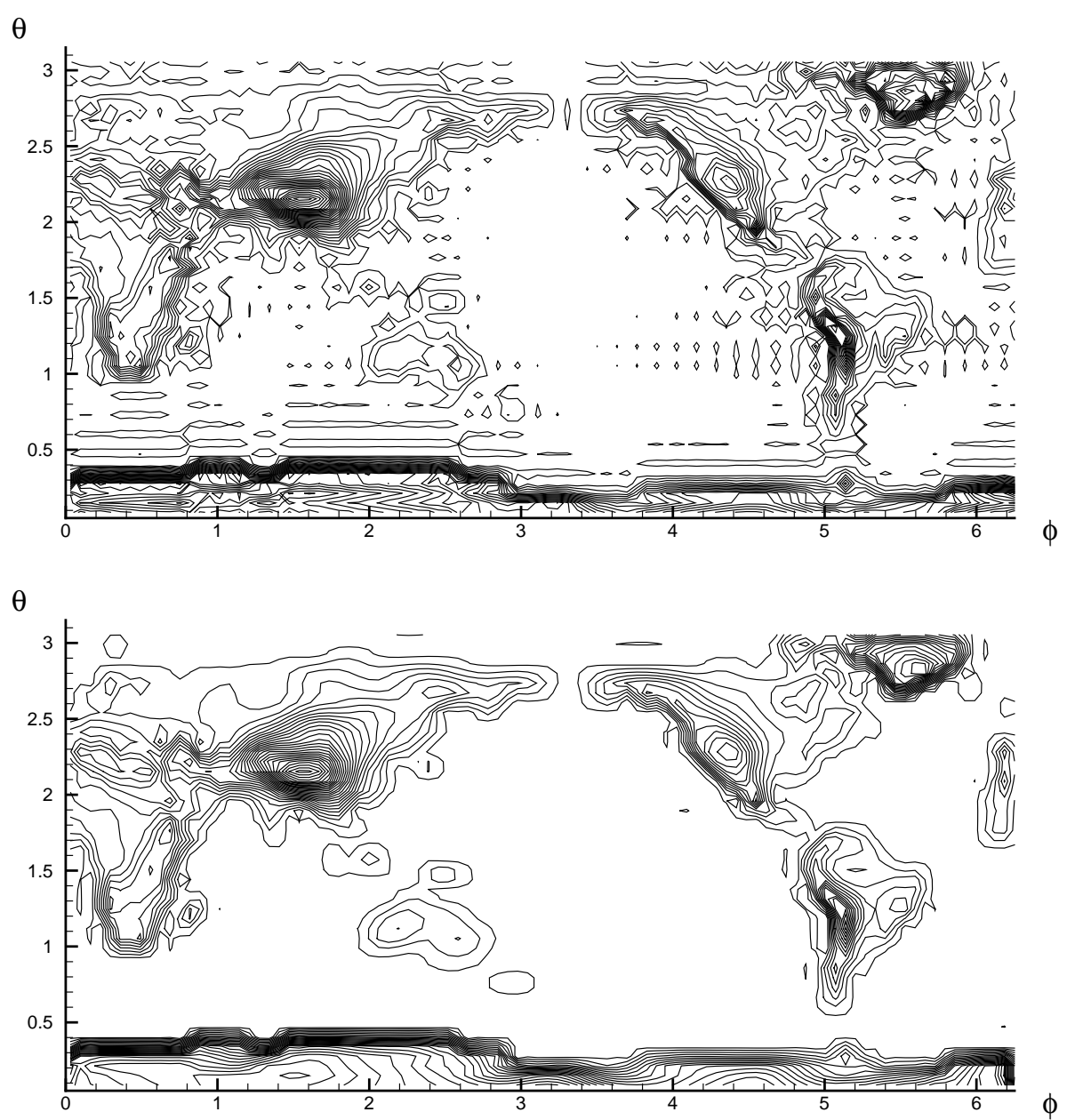

FiguRE 5.9. The reconstruction of the earth's topographical data using (a) the regular spectral approximation and (b) the localized regularization method.

- Edge detection for functions with discontinuities in the derivatives. Since spectral accuracy is linked to global smoothness, determining the jumps in the derivative of a function is critical to retain high accuracy in the reconstruction method.

Acknowledgements. Research was supported in part by NSF Grant No. DMS01-07428 (E.T. and A.G.), the Sloan Foundation (A.G.) and ONR Grant No. N00014-1-J-1076 (E.T.).

\section{REFERENCES}

[1] N.S. Banerjee and J. Geer, Exponential approximations using Fourier series partial sums, ICASE Report No. 97-56, NASA Langley Research Center (1997).

[2] N. Bary, Treatise of Trigonometric Series. The Macmillan Company, New York (1964).

[3] H.S. Carslaw, Introduction to the Theory of Fourier's Series and Integrals. Dover (1950).

[4] K.S. Eckhoff, Accurate reconstructions of functions of finite regularity from truncated series expansions. Math. Comp. 64 (1995) 671-690.

[5] K.S. Eckhoff, On a high order numerical method for functions with singularities. Math. Comp. 67 (1998) 1063-1087.

[6] A. Gelb and E. Tadmor, Detection of edges in spectral data. Appl. Comput. Harmon. Anal. 7 (1999) 101-135. 
[7] A. Gelb and E. Tadmor, Detection of edges in spectral data. II. Nonlinear Enhancement. SIAM J. Numer. Anal. 38 (2001) 1389-1408.

[8] B.I. Golubov, Determination of the jump of a function of bounded p-variation by its Fourier series. Math. Notes 12 (1972) 444-449.

[9] D. Gottlieb and C.-W. Shu, On the Gibbs phenomenon and its resolution. SIAM Rev. (1997).

[10] D. Gottlieb and E. Tadmor, Recovering pointwise values of discontinuous data within spectral accuracy, in Progress and Supercomputing in Computational Fluid Dynamics, Proceedings of a 1984 U.S.-Israel Workshop, Progress in Scientific Computing, Vol. 6, E.M. Murman and S.S. Abarbanel Eds., Birkhauser, Boston (1985) 357-375.

[11] G. Kvernadze, Determination of the jump of a bounded function by its Fourier series. J. Approx. Theory 92 (1998) $167-190$.

[12] E. Tadmor and J. Tanner, Adaptive mollifiers for high resolution recovery of piecewise smooth data from its spectral information, Foundations of Comput. Math. Online publication DOI: 10.1007/s002080010019 (2001), in press.

[13] A. Zygmund, Trigonometric Series. Cambridge University Press (1959).

To access this journal online:

www.edpsciences.org 